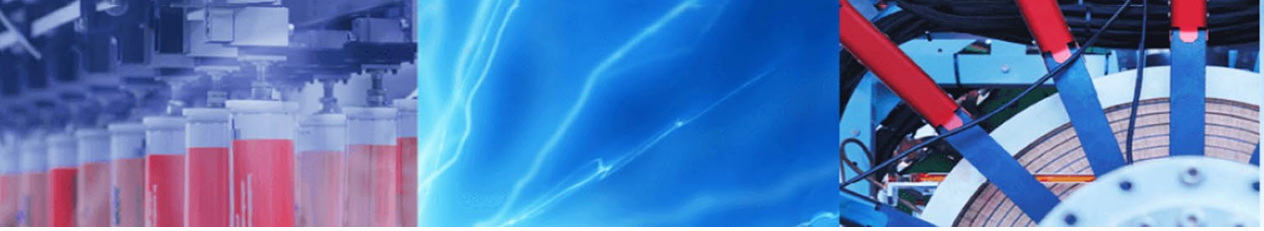

Research Article

\title{
Verification, enhancement and mathematical analysis of EBG structure using complex geomsetrical shapes and eigenmode analysis approach
}

\author{
Rajshri C. Mahajan ${ }^{1}$ (D) Vibha Vyas ${ }^{1}$ (D)
}

Received: 28 August 2019 / Accepted: 28 November 2019 / Published online: 5 December 2019

(c) Springer Nature Switzerland AG 2019

\begin{abstract}
The study presents the enhancement in analysis formulae and its verification for Electromagnetic Band Gap (EBG) structure using the Eigenmode analysis method. Eigenmode analysis of EBG structure is a compact and prolific method for obtaining its performance parameters like surface bandgap (bandwidth) and resonant frequency. The enhancement in mathematical expressions of the gap width, the capacitance, and the bandwidth is carried out using the simple geometrical shapes like square, circle, and hexagon. The verification of the enhanced formulae of the EBG structure is conducted using complex shapes. The theoretical and simulated results agree with each other up to $94 \%$ accuracy.
\end{abstract}

Keywords Unit cell · EBG structure · Surface bandgap

\section{Introduction}

In the recent past, microstrip antenna with EBG structure was popular due to its peculiar features like surface wave suppression, bandwidth enhancement, and improvement in the efficiency of antenna [1-5]. The characterization of EBG structure is obtained using techniques like planewave-expansion method [6-8], finite-difference method [9], finite-element method [10, 11], and transfer-matrix method [12, 13]. A variety of other techniques are also used, such as the effective medium theory [14], phasedarray method [15], Eigenmode expansion method [16], array scanning method [17].], and hybrid methods [18, 19], Full-wave simulation, Finite Domain Time Difference (FDTD) method and circuit model-based method [20]. The full-wave simulation method based on a diffraction grating is developed for a finite number of periodical layers of the EBG structure [21].

Generalized-pencil-of-function (GPOF) algorithm is developed as a post-processing procedure in the finitedifference time-domain (FDTD) method for EBG structure analysis. The technique reduces the computational burden by reducing an excessive number of time steps [22]. The circuit model is build up using the patches and meander lines as connecting bridges based on the rigorous analysis of the propagation effects for each unit cell of the EBG structure [23].

The circuit model for the hybrid structure consisting of resistance, which represents the permeability losses due to the ferrite layer is proposed. It is also demonstrated that both the inductor and resistor components are frequencydependent [24].

The research of any technology relies on mathematical modeling and reaches up-to computational simulation. In this paper, the computational simulation method is used to enhance the basic analysis formulae. The FDTD based Eigenmode analysis is used to simulate the unit cells of various shapes of the EBG structure. These simulated results are put into a mathematical model, and the enhancement of formulae like the gap width, capacitance and surface bandgap are achieved.

The major contributions of this paper are focused on,

Rajshri C. Mahajan, mrc.extc@coep.ac.in | ${ }^{1}$ Department of Electronics and Telecommunication, Government College of Engineering, Pune (COEP), Pune, Maharashtra 411005, India. 
- Investigation and validation of the expressions of the capacitance and bandwidth for considered geometrical shapes like circle, hexagon, and complex designs like circular fractal shapes where the gap-width is not constant at every point on the edge of cells.

- The analysis is carried out using the Eigenmode analysis approach, and the expressions of bandwidth and capacitance are validated and enhanced.

The paper organization is as follows. In Sect. 2, a model of EBG structure and its mathematical analysis is demonstrated. Section 3 discusses the Eigenmode analysis approach using the simulation setup for EBG analysis. Section 4 focuses on simulations of various shapes, while Sect. 5 elaborates on results, and suggestions regarding the enhancements in the analysis of the bandwidth expressions. Section 6 describes the brief conclusions.

\section{EBG structure and its mathematical analysis}

EBG structure is a modified ground surface with the metallic patches printed on the substrate. These patches are known as cells which are either periodic or non-periodic. These cells are separated by a particular gap width [25-28]. The dimensions of a patch and gap width are much less than the operating frequency $(\lambda)$ of an antenna [3, 29-31]. The EBG cells are shorted to the ground surface using conducting pins or wires, which are called vias [32-34]. The geometry of EBG structure, typically Sivenpipper's Mushroom structure, is shown in Fig. 1.

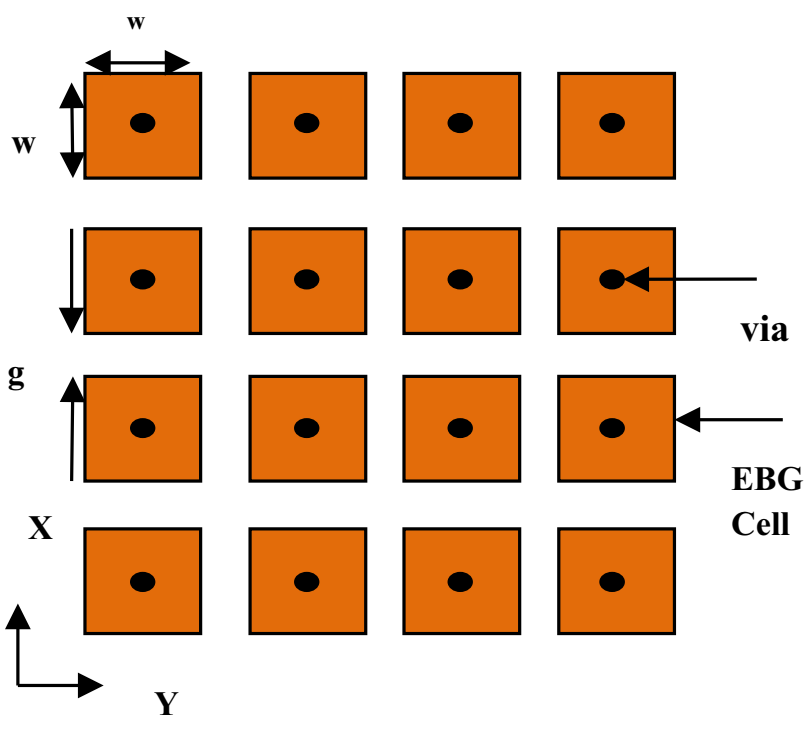

Fig. 1 Geometry of Mushroom like Sivenpiper's EBG structure
A Sivenpipper's mushroom EBG cell comprises a metallic patch of width ' $w$ ' which is separated from another patch by the distance ' $g$ ', which is called gap width. The via is present between EBG cell and ground surface, and its height is ' $h$.'The patch width of EBG cell, gap width, and height of via form the effective capacitance and inductance $[20,32,35,36]$. Figure 2 shows the side view of mushroom, like Sivenpipper's EBG structure showing the dimensions of the EBG structure.

$L C$ resonating circuit model best describes the structure, and its effective capacitance and inductance values are evaluated with the help of Eqs. (1) and (2),

$C=\frac{w \varepsilon_{0}\left(1+\varepsilon_{r}\right)}{\pi} \cosh ^{-1}\left(\frac{w+g}{g}\right)$

$L=\mu h$

where ' $w$ ' is a unit cell patch width, ' $\varepsilon_{0}$ ' is free space permittivity, ' ${ }^{\prime}{ }_{r}^{\prime}$ is relative permittivity of the substrate, ' $g$ ' is a gap width, ' $\mu_{r}{ }^{\prime}$ is the relative permeability of the substrate, ' $\mu_{0}{ }^{\prime}$ is free space permeability $\left(\mu=\mu_{0} \mu_{r}\right)$, and ' $h$ ' is the height of the substrate. The physical parameters of the EBG cell are shown in Fig. 3a. The conducting via has the height of $h$, and its radius is r. Figure $3 \mathrm{~b}$ shows the equivalent capacitance ' $C$ ' and inductance ' $L$.'

The EBG structure's for the calculation of bandwidth, resonant frequency, and impedance of EBG structure are derived as follows $[20,32,36]$.

Let $Z_{S}$ be the surface impedance of the EBG structure. The reflection coefficient $\Gamma$ and the reflection phase can be computed as:

$Z_{S}=j X$

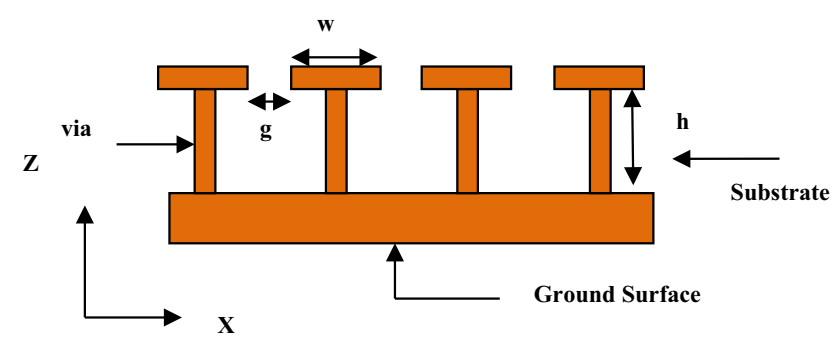

Fig. 2 Mushroom-like electromagnetic bandgap (EBG) structure's side view

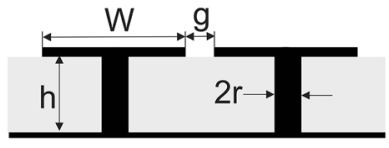

(a)

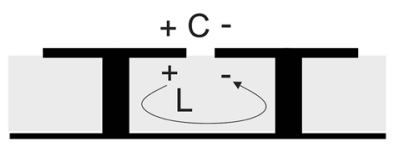

(b)
Fig. 3 a Physical parameters of the EBG cell and $\mathbf{b}$ Equivalent LC model

\section{SN Applied Sciences}


$\Gamma=\frac{\left(Z_{S}-\eta_{0}\right)}{\left(Z_{S}+\eta_{0}\right)}$

$|\Gamma|=1$

$\angle \Gamma=\pi-2 \tan ^{-1}\left(\frac{x}{\eta_{0}}\right)$

The bandwidth of the EBG structure is defined as the band of frequencies where the reflection phase is between $+90^{\circ}$ and $-90^{\circ}$. The condition of the surface bandwidth can be found as:

$-90^{\circ} \leq \angle \Gamma \leq+90^{\circ}$

Using Eqs. 6 and 7, it can be written as,

$-\eta_{0} \geq X \geq+\eta_{0}$

Let $\omega_{1}$ be the angular frequency where $X=\eta_{0}$, so

$Z_{s}=j \eta_{0}$

Further,

$Z_{S}=\frac{j \omega_{1} L}{1-\left(\frac{\omega_{1}}{\omega_{0}}\right)^{2}}$

$\therefore \omega_{0}^{2}-\omega_{1}^{2}=\frac{\left(\omega_{0}^{2} L \omega_{1}\right)}{\eta_{0}}$

Let $\omega_{2}$ be the angular frequency when $X=-\eta_{0}$, From Eqs. (10) and (11),

$B W=\omega_{2}-\omega_{1}=\frac{\omega_{0}^{2} L}{\eta_{0}}=\frac{1}{120 \pi} \sqrt{\frac{L}{C}}$

From Eq. (12), it can be observed that the bandwidth given in the relevant literature is equal to the mathematical expression derived [36].

The equations from (1)-(12) are used to find the performance parameters of the EBG structure like bandwidth, resonant frequency, and the surface impedance. These equations are derived for a square-shaped EBG cell where the gap width ' $\mathrm{g}$ ' is constant between adjacent cells.

\section{Eigenmode approach for EBG Structure}

The EBG structure is analyzed using full-wave simulation methods like Finite Element Method (FEM) and Method of Moments (MOM). The full-wave simulation methods require huge memory and time for their execution.
Finite Difference Time Domain (FDTD)-Periodic Boundary Condition (PBC) is widely used to analyze the EBG structure. It gives a wide visibility of performance using a simple simulation setup $[37,38]$. The method helps to predict the performance of the EBG structure by simulating a single cell of the EBG structure. Figure 4 depicts the simulation setup of Eigenmode analysis.

FDTD-PBC based unit cell analysis method is carried out using two approaches viz. scattering mode analysis and Eigenmode analysis. For the first one, an EBG cell is placed with an airbox of finite size, the sidewalls of the air box are applied with Periodic Boundary Condition (PBC). The upper surface of the air box is applied with a floquet port. The scattering analysis gives a reflection and transmission curves for the selected frequency range, and the surface band is predicted.

For Eigenmode analysis, the EBG cell is placed in the airbox of a height six times more than the height of the substrate. A Perfectly Matched Layer (PML) boundary is applied to terminate the airbox. This airbox is enclosed in another airbox whose sidewalls are applied with PBC along $x$ and $y$ directions. The phase shift along the transverse direction is kept at $0^{\circ}$, while the phase along the direction of propagation is varied from $0^{\circ}$ to $180^{\circ}$.

The dispersion curve and the surface band gap for various modes obtained using Eigenmode analysis and a graphical method $[39,40]$.

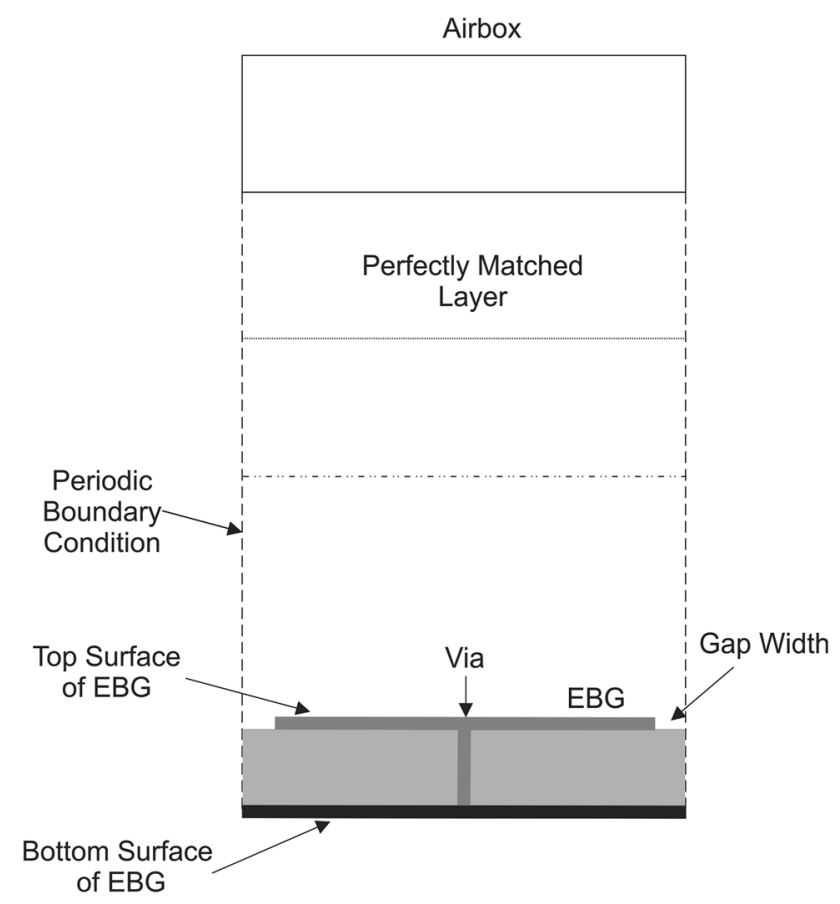

Fig. 4 Simulation Setup design of FDTD/PBC based Eigenmode analysis 
The following steps are carried out for analysis of EBG structure using the FDTD-PBC method wherein the electromagnetic fields are calculated using the following technique [20].

- The electromagnetic (EM) fields in the interior of the EBG cell in free space are updated Yee's scheme.

- Equations (13) and (14) are used to calculate EM fields on the periodic boundaries of a computational domain.

$$
\begin{aligned}
& E(x=0, y, z, t)=E(x=p, y, z, t) e^{j p k_{x}} \\
& H(x=0, y, z, t)=H(x=p, y, z, t) e^{j p k_{x}}
\end{aligned}
$$

where the periodicity of EBG cells is $p$.

- The elimination of the reflections in the Perfectly Matched Layer (PML) region is possible by setting the material properties as per (15) (16), (17), and (18).

$\overline{\bar{s}}=\left[\begin{array}{ccc}s_{x}^{-1} & 0 & 0 \\ 0 & s_{x} & 0 \\ 0 & 0 & s_{x}\end{array}\right] \cdot\left[\begin{array}{ccc}s_{y} & 0 & 0 \\ 0 & s_{y}^{-1} & 0 \\ 0 & 0 & s_{y}\end{array}\right] \cdot\left[\begin{array}{ccc}s_{z} & 0 & 0 \\ 0 & s_{z} & 0 \\ 0 & 0 & s_{z}^{-1}\end{array}\right]$

$s_{x}=1+\frac{\sigma_{x}}{j \omega \varepsilon_{0}}$

$s_{y}=1+\frac{\sigma_{y}}{j \omega \varepsilon_{0}}$

$$
\left(s_{z}=1+\frac{\sigma_{z}}{j \omega \varepsilon_{0}}\right)
$$

The following procedure is carried out to implement a constant $k_{x}$ method.

- The propagation constant $k_{x}(\beta)$ is obtained.

- Acquired results are plotted in $k_{x}$-frequency plane.

- The Eigen frequencies are identified for the propagation constants in $\mathrm{x}$ and $\mathrm{y}$ directions.

- The extraction of the resonant frequencies of surface waves is undertaken.

- The Dispersion is plotted to obtain the surface band gap $[20,41]$.

In this research work, Eigenmode analysis approach is applied to obtain the bandwidth and the center frequency of the unit EBG cell. This empirical bandwidth is verified using a mathematical approach, and an enhancement in its formulation is suggested for an improvement in results.

\section{Simulation of experiments for various shapes of EBG cell}

In this section, the simulations are carried out for the basic geometrical shapes using Eigenmode analysis. The simulations are conducted using High-Frequency Simulation Software version 11 (HFSS 11). The EBG cells of the same footprint and gap widths are taken into consideration. The effect of gap width for complex shapes and its expression is enhanced using fractal shapes.

$$
\mathrm{g}=1 \mathrm{~mm}
$$

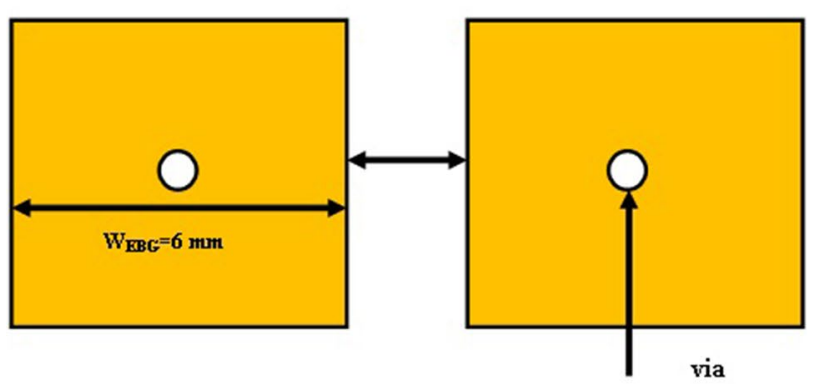

(a)

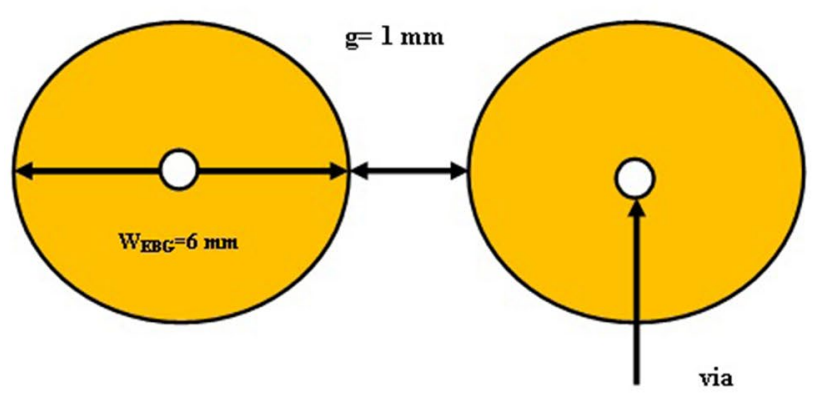

(b)

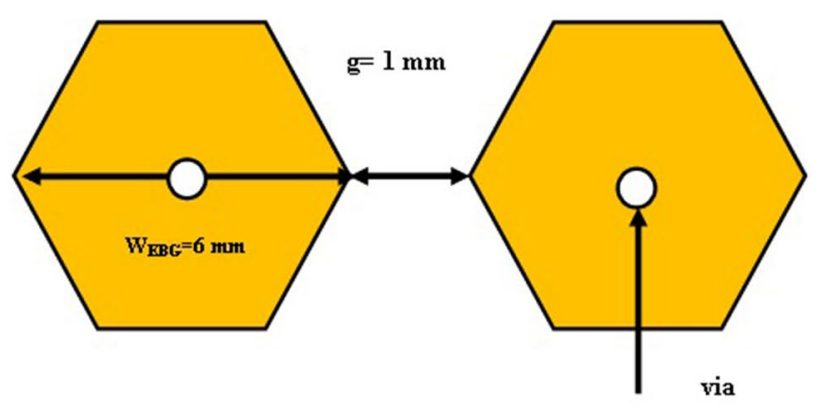

(c)

Fig. 5 Dimensions of a Square, $\mathbf{b}$ Circular and $\mathbf{c}$ Hexagonal unit cell for Eigen Analysis 
Initially, the square-shaped EBG cell is simulated using the dimensions as $\mathrm{w}=6 \mathrm{~mm}, \mathrm{~h}=1.6 \mathrm{~mm}, \mathrm{~g}=1 \mathrm{~mm}, \varepsilon_{\mathrm{r}}=4.4$, $r=0.1 \mathrm{~mm}$. The geometry and dimensions of the squareshaped EBG cell is shown in Fig. 5a. For circle and hexagonal shapes, the width or footprint of the cell is kept as $\mathrm{w}_{\mathrm{EBG}}=6 \mathrm{~mm}, \mathrm{~h}=1.6 \mathrm{~mm}, \varepsilon_{\mathrm{r}}=4.4, \mathrm{r}=0.1 \mathrm{~mm}$. The gap width $\mathrm{g}$, for circular and hexagonal shapes, is considered between the two nearest points of adjacent EBG cells, as shown in Fig. $5 \mathrm{~b}$ and c.

Table 1 describes the bandwidths and center frequencies obtained using Eigenmode analysis. From Table 1, it is observed that though the dimensions of the EBG cells are the same and the corresponding bandwidths and center frequencies are different for each case. This variation is due to the shape of the EBG cell, which offers different gap width values between the adjacent cells.

It is observed from Eqs. (1) and (12) that the gap width plays a vital role in deciding the capacitance of the EBG structure as well as its bandwidth.

To study and observe the effect of the gap width for circle and hexagon shape, the gap widths between them are measured at different points, as shown in Figs. 6 and 7. The gap widths $g_{1}$ and $g_{2}$ are considered between the two nearest points and farthest points, respectively, for these two shapes. There are an infinite number of values of gap width between $g_{1}$ and $g_{2}$.

Therefore, for accurate analysis of such complex shapes, there is a need to modify the gap width equation. Various gap widths should be considered between the adjacent cells. The gap width for such shapes is selected in such a way that the average gap width is found to be equal to $1 \mathrm{~mm}$ for the verification of practical and simulated results.

Exhaustive computational simulations are carried out by varying the gap width for the validation of the equation. The equivalent gap width is calculated using averaging of discrete samples of the available gap widths as described in Eq. (19),

$g_{\text {avg }}=\frac{1}{n} \sum_{n=1}^{n} g_{n}$

Equation (19) is validated for various sizes of circular and hexagonal shapes. To further investigate the performance

Table 1 Comparison of band-gaps and center frequencies for three basic shapes

\begin{tabular}{lllll}
\hline $\begin{array}{l}\text { Shape of unit } \\
\text { cell }\end{array}$ & $\begin{array}{l}\text { Width of } \\
\text { patch w } \\
(\mathrm{mm})\end{array}$ & $\begin{array}{l}\text { Gap } \\
\text { width g } \\
(\mathrm{mm})\end{array}$ & $\begin{array}{l}\text { Surface } \\
\text { band-gap } \\
(\mathrm{GHz})\end{array}$ & $\begin{array}{l}\text { Center } \\
\text { frequency } f_{r} \\
(\mathrm{GHz})\end{array}$ \\
\hline Square & 6 & 1 & $1.83-3.26$ & 2.545 \\
Circle & 6 & 1 & $2.08-4.83$ & 3.455 \\
Hexagon & 6 & 1 & $2.11-4.72$ & 3.415 \\
\hline
\end{tabular}

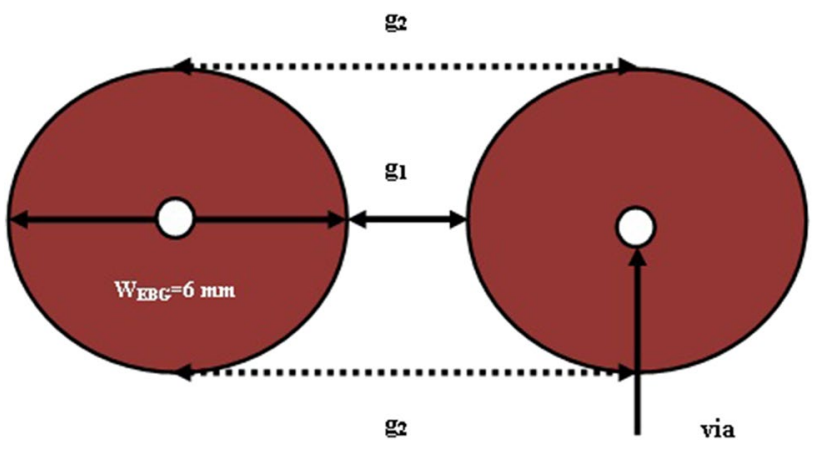

Fig. 6 Geometry of Circular EBG cell and its corresponding dimensions

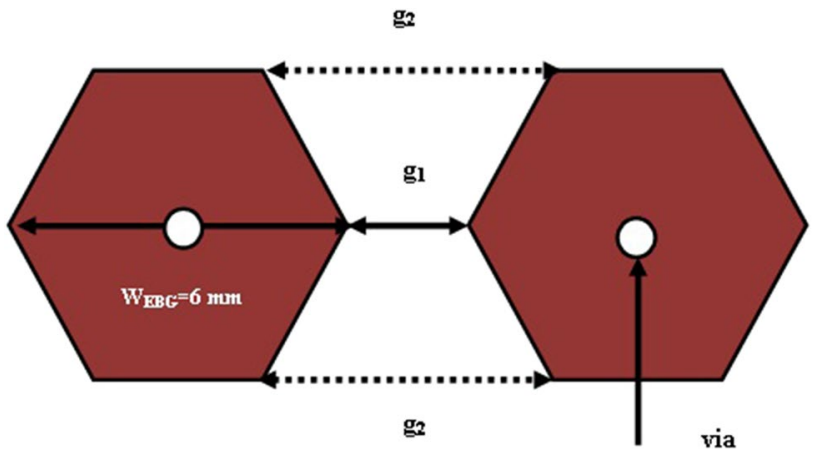

Fig. 7 Geometry of Hexagonal EBG cell and its corresponding dimensions

of this equation, complex shapes with more number of intermediate gap widths are designed, simulated, and tested. Figure 8 shows the circular fractal shaped EBG cell with one iteration where the width or footprint of the cell is kept $6 \mathrm{~mm}$ constant throughout. The gap widths are different at different points but are adjusted to the values such that the average gap width, $g_{\text {avg, }}$ is equal to $1 \mathrm{~mm}$.

Two iterations based circular fractal shaped EBG cell is designed having a footprint size of $6 \mathrm{~mm}$ and simulated using Eigenmode analysis approach.

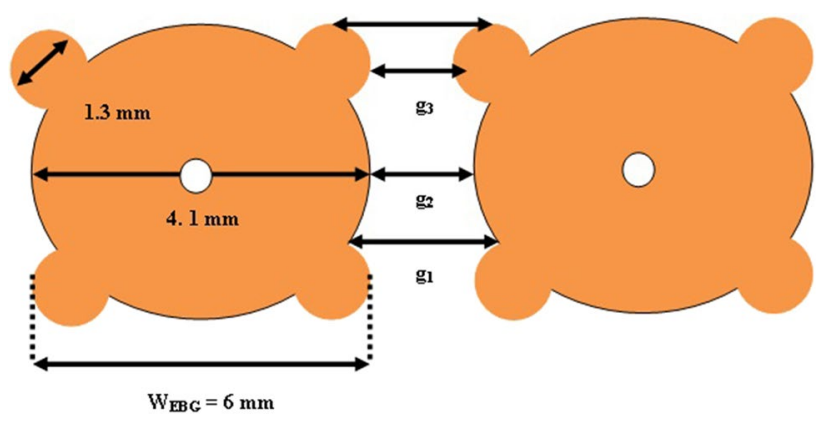

Fig. 8 Geometry of circular-shaped fractal unit cell with one iteration 


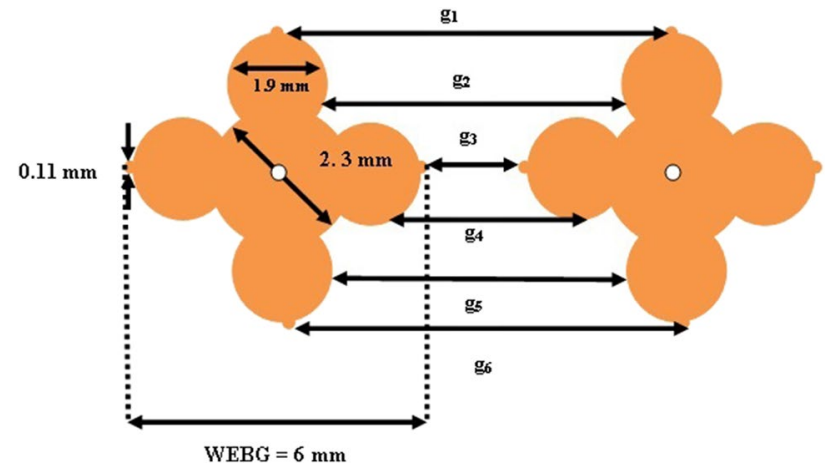

Fig. 9 Geometry of circular-shaped fractal unit cell with 2 iterations

Figure 9 shows the geometry and dimensions of a circular-shaped fractal unit cell with two iterations.The parameters obtained after simulation are tabulated in Table 2.

Table 2 clearly states that even for the same width of patch and gap-width, variation in the parameters for various shapes is obtained. The gap-width is kept as $1 \mathrm{~mm}$ in all cases, but for square shape, it was constant for every point on adjacent edges. The gap width for other shapes is not the same throughout the curvature. It is, therefore, needed to enhance the theoretical expression for capacitance as it depends on a gap-width.

\section{Discussions on the results}

This section focuses on the need for improvement in bandwidth expression of the EBG structure. The improvement is suggested based on the simulation results of fractal shapes of EBG cells obtained using FDTD-PBC analysis method.

The enhanced capacitance expression can be given as per Eq. (20),

$C=\frac{w \varepsilon_{0}\left(1+\varepsilon_{r}\right)}{\pi} \cosh ^{-1}\left(\frac{w+g_{\text {avg }}}{g_{\text {avg }}}\right)$

The following steps are carried out for investigating the validity of bandwidth expression.

- Mathematical analysis is carried out considering the square-shaped EBG cell with various patch widths and gap widths.

- The corresponding equivalent, capacitance, inductance, and bandwidth are calculated.

Several iterations of simulations are conducted to obtain the modified expression for the bandwidth. The simulations are conducted for various gap widths and patch widths of the EBG cells. The results of a few of the simulations are tabulated in Table 3. The theoretical as well as simulated results obtained for square-shaped unit cells for various widths
Table 2 Parameters for circular fractal shaped EBG cell

\begin{tabular}{lllcl}
\hline Shape of unit cell of fractal shape & $\begin{array}{l}\text { Width of patch } \\
w(\mathrm{~mm})\end{array}$ & $\begin{array}{l}\text { Gap width } \\
\mathrm{g}_{\mathrm{avg}}(\mathrm{mm})\end{array}$ & $\begin{array}{l}\text { Surface band- } \\
\text { gap }(\mathrm{GHz})\end{array}$ & $\begin{array}{l}\text { Center } \\
\text { frequency } f_{r} \\
(\mathrm{GHz})\end{array}$ \\
\hline Circle & 6 & 1 & $2.11-4.72$ & 3.415 \\
Circle with 1st iteration & 6 & 1 & $1.8-5.21$ & 3.505 \\
Circle with 2nd iteration & 6 & 1 & $1.1-6.32$ & 3.71 \\
\hline
\end{tabular}

Table 3 Theoretical and practical values of parameters of square-shaped EBG structure

\begin{tabular}{lllllll}
\hline $\begin{array}{l}\text { Patch width } \\
W(\mathrm{~mm})\end{array}$ & $\begin{array}{l}\text { Height of sub- } \\
\text { strate } h(\mathrm{~mm})\end{array}$ & $\begin{array}{l}\text { Gap width } G \\
(\mathrm{~mm})\end{array}$ & $\begin{array}{l}\text { Theoretical induct- } \\
\text { ance } L(\mathrm{nH})\end{array}$ & $\begin{array}{l}\text { Theoretical } \\
\text { inductance } C(\mathrm{pF})\end{array}$ & $\begin{array}{l}\text { Theoretical Band- } \\
\text { width BW }(\mathrm{Hz})\end{array}$ & $\begin{array}{l}\text { Band-gap using Eigen- } \\
\text { mode analysis approach } \\
(\mathrm{GHz})\end{array}$ \\
\hline 4 & 1.6 & 0.5 & 2.0112 & 0.162827 & 0.294953146 & 2.9801 \\
4 & 1.6 & 1 & 2.0112 & 0.129281 & 0.331015708 & 4.4345 \\
4 & 1.6 & 2 & 2.0112 & 0.09941 & 0.377486817 & 7.1025 \\
6 & 1.6 & 0.5 & 2.0112 & 0.275485 & 0.226760828 & 1.2043 \\
6 & 1.6 & 1 & 2.0112 & 0.222809 & 0.252144925 & 1.6559 \\
6 & 1.6 & 2 & 2.0112 & 0.000169179 & 9.150464168 & 701441.9 \\
8 & 1.6 & 0.5 & 2.0112 & 0.0000563929 & 15.84910154 & 5062117.6 \\
8 & 1.6 & 1 & 2.0112 & 0.000112785 & 11.20703169 & 146948.3 \\
8 & 1.6 & 2 & 2.0112 & 0.000225568 & 7.924605745 & 417133.7 \\
\hline
\end{tabular}


Table 4 Theoretical and practical values of parameters of complex shapes of EBG

\begin{tabular}{llll}
\hline Shape of unit cell & $\begin{array}{l}\text { Width of } \\
\text { patch w }(\mathrm{mm})\end{array}$ & $\begin{array}{l}\text { Surface band-gap } \\
\text { bandwidth }(\mathrm{GHz})\end{array}$ & $\begin{array}{l}\text { Calculated bandwidth using } \\
\text { enhanced formula in } \mathrm{GHz}\end{array}$ \\
\hline Square & 6 & 1.48 & 1.4692 \\
Hexagon & 6 & 2.75 & 2.6987 \\
Circle & 6 & 2.61 & 2.581 \\
Fractal circle with 1st iteration & 6 & 3.41 & 3.395 \\
Fractal circle with 2nd iteration & 6 & 5.22 & 5.183 \\
\hline
\end{tabular}

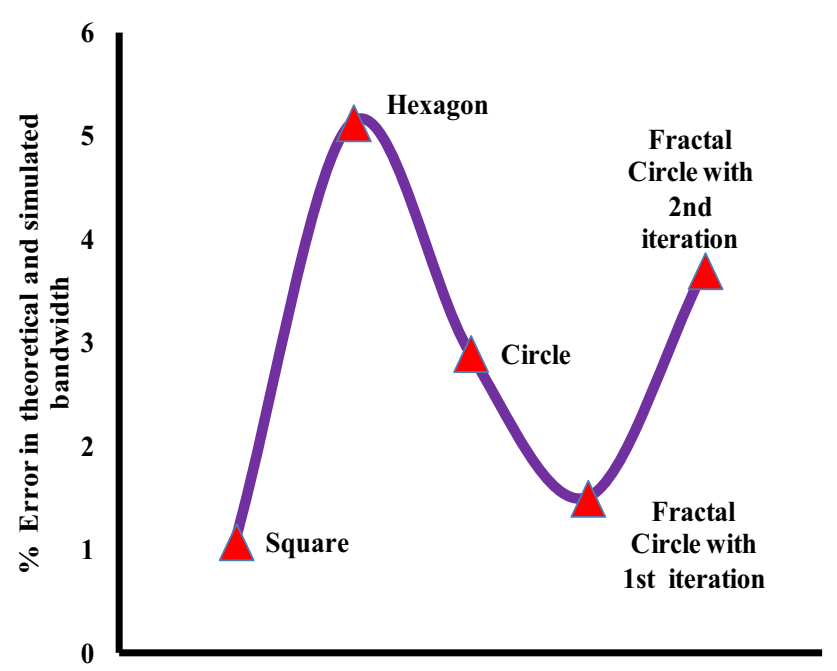

Fig. 10 Plot of error in simulated and calculated results for various shapes

of patch and gap widths are shown in Table 3. It can be observed that there is a remarkable difference in theoretical and simulated bandwidth results. Hence, there is a requirement to modify the formula for bandwidth. The enhanced expression for bandwidth which is stated in Eq. (21),

$B W=\frac{L^{1.5}}{\pi C^{1.8}}$

The Eq. (21) is formalized using regression analysis and based on an empirical method. The validation is carried out for circle, hexagon, and circular fractal shapes, and the results are tabulated in Table 4.

From Table 4, it is observed that simulated and calculated bandwidths agree with each other up to $94 \%$ accuracy. Figure 10 shows the plot of percentage error in theoretical and practical results concerning the shapes of EBG cells.

The strong agreement between the simulated and calculated results (obtained from the enhanced formula of bandwidth) is the achievement of the research. The earlier research has focused on the new techniques of EBG structure analysis. While this paper discusses enhancing the traditional analysis formulae so that the accurate mathematical analysis can be carried out.

\section{Conclusion}

The modern tools for the analysis of complex designs of microstrip antenna and EBG structure are useful for the prediction of their performance. The Eigenmode based analysis method proves to be efficient for the verification of mathematical analysis and enhancement of expressions used for the analysis. The gap width for some geometrical shapes

like circular and hexagonal unit cells is not uniform at every point between the adjacent cells. Therefore, an improvement in gap-width calculation based on sampling and averaging method is suggested. This improvement is validated with practical results obtained using FDTD-PBC based Eigenmode analysis approach. The equation for gap width is verified for complex shapes based on circular fractal shapes of EBG cells. Further, the analytical formulation of capacitance is enhanced using regression analysis and verified with simulated results. The Eigenmode analysis can be further investigated for exploring different parameters of EBG structure and similar materials like Artificial Magnetic Materials (AMC), High Impedance Surface (HIS), Photonic Band Gap (PBG) materials.

Funding There is no funding from any agency for the research work proposed in the Paper.

\section{Compliance with ethical standards}

Conflict of interest The authors declare that they have no conflict of interest.

\section{References}

1. Garg R (2001) Microstrip antenna design handbook, ISBN13978-0-521-88991-9, Artech House, Inc

2. Kumar G, Ray KP (2003) Broadband microstrip antennas', ISBN1-58053-244-6, Artech House, Inc

3. Weiss SJ, Kim IK, Wang H, Varadan VV (2012) Embedded wideband met resonator antenna on a high-impedance ground plane for vehicular applications. IEEE Trans Veh Technol 61(4):1665-1672

4. Winn JN, Meade RD, Joannopoulos JD (1995) Photonic Crystals. Princeton University Press, Princeton 
5. Yablonovitch E (1993) Photonic band-gap structure. J Opt Soc Am B OSA Publ 10:283-295

6. Joannopoulos JD, Meade RD, Winn JN (2008) Photonic crystals: molding the flow of light, 2nd edn. Princeton University Press

7. Ho KM, Chan CT, Soukoulis CM (1990) Existence of a photonic bandgap in periodic dielectric structures. Phys Rev Lett 65:3152-3155

8. Munk BA, Burrell GA (1979) Plane-wave expansion for arrays of arbitrarily oriented piecewise linear elements and its application in determining the impedance of a single linear antenna in a lossy half-space. IEEE Trans Antennas Propag 27:331-343

9. Yang HYD (1996) Finite-difference analysis of 2-D photonic crystals. IEEE Trans Microw Theory Tech 44(12):2688-2695

10. Coccioli R, Itoh T, Pelosi G (1997) A finite element-generalized network analysis of finite thickness photonic crystals. In: IEEE MTT-S International Microwave Symposium Digest, pp 195-198

11. Pelosi G, Cocchi A, Monorchio A (2000) A hybrid FEM-based procedure for the scattering from photonic crystals illuminated by a Gaussian beam. IEEE Trans Antennas Propag 48(6):973-980

12. Pendry JB, MacKinnon A (1992) Calculation of photon dispersion relations. Phys Rev Lett 69(9):2772-2775

13. Pendry JB (1994) Photonic structures. J Mod Opt 41(2):209-229

14. Lalanne $P$ (1996) Effective medium theory applied to photonic crystals composed of cubic or square cylinders. OSA Publ J Appl Opt 35(27):5369-5380

15. Caloz C, Skrivervik AK, Gardiol E (2002) An efficient method to determine Green's functions of a two-dimensional photonic crystal excited by a line source-The phased-array method. IEEE Trans Microw Theory Tech 50(5):1380-1391

16. Yang H-YD (1999) Theory of antenna radiation from photonic band-gap materials. Electromagnetics 19(3):255-276

17. Suzuki T, Yu PKL (1995) Emission power of an electric dipole in the photonic band structure of the FCC lattice. OSA Publ J Opt Soc Am B 12(4):570-582

18. Gedney SD, Lee JF, Mittra R (1992) A combined FEM/MoM approach to analyze the plane wave diffraction by arbitrary gratings. IEEE Trans Antennas Propag 40:363-370

19. Lucas EW, Fontana TP (1995) A 3-D hybrid finite element/boundary element method for the unified radiation and scattering analysis of general infinite periodic arrays. IEEE Trans Antennas Propag 43(2):145-153

20. Yang F, Rahmat $Y$ (2009) Electromagnetic band gap structures in antenna engineering. Cambridge University Press, Cambridge

21. Frezza F, Pajewski L, Schettini G (2003) Characterization and design of two-dimensional electromagnetic band-gap structures by use of a full-wave method for diffraction gratings. IEEE Trans Microw Theory Tech 51(3):941-951

22. Lalas AX, Kriezis EE, Tsiboukis TD (2009) Accelerating FDTD simulations for Eigen frequency detection in EBG devices by means of the GPOF method. IEEE Trans Magn 45(3):1316-1319

23. Mohajer-Iravani B, Ramahi OM (2010) Wideband circuit model for planar EBG structures. IEEE Trans Adv Packag 33(1):169-179

24. Bell JM, Iskander MF (2008) Equivalent circuit model of an ultrawideband hybrid EBG/Ferrite structure. IEEE Antennas Wirel Propag Lett 7:573-576

25. Deo P, Mehta A, Massey PJ, Nakano H, Mirshekar-Syahkal D (2010) Thickness reduction and performance enhancement of steerable square loop antenna using hybrid high impedance surface. IEEE Trans Antennas Propag 58(5):1477-1485

26. Hassam MAM, Kishk AA (2017) Bandwidth study of the Stacked Mushroom EBG unit cell. IEEE Trans Antennas Propag 65(8):4357-4362
27. Naidu PV, Kumar A, Rajkumar R (2018) Design, analysis, and fabrication of compact dual-band uniplanar meandered ACS fed antenna for $2.5 / 5 \mathrm{GHz}$ applications. Microsystems Technologies 25(1):1-8

28. Zoubiri B, Mayouf A, Mayouf F, Abdelkebir S, Devers T (2018) Enhancement of front to back ratio and gain of rectangular microstrip antenna using novel elliptical EBG structure. Microsyst Technol 24(8):3241-3244

29. Costa F, Monorchio A, Genovesi S (2013) A chipless RFID based on multiresonant high-impedance surfaces. IEEE Trans Microw Theory Tech 61(1):146-153

30. Hao Y, Wen D, Munoz MO (2018) A compact and low-profile MIMO antenna using a miniature circular high-impedance surface for wearable applications. IEEE Tran Antennas Propag 66(1):96-104

31. Vallecchi A, De Luis JR, Capolino F (2012) Low profile fully planar folded dipole antenna on a high impedance surface. IEEE Trans Antennas Propag 60(1):51-62

32. Sievenpiper D, Zhang L, Broas J, Alexopolous NG, Yablonovitch $E$ (1999) High-impedance electromagnetic surfaces with a forbidden frequency band. IEEE Trans Microw Theory Tech 47(11):2059-2074

33. Zhu C, Chen X, Li L, Liang CH, Su ZJ (2012) Dual-band high impedance surface with mushroom-type cells loaded by symmetric meandered slots. IEEE Trans Antennas Propag 60(10):4677-4687

34. Mahajan RC, Vyas V (2019) Wine glass shaped microstrip antenna with woodpile structure for wireless applications. Majl J Electr Eng 13(1):37-44

35. Lafmajani IA, Rezaei P (2012) A novel frequency-selective metamaterial to improve helix antenna. Front Inf Technol Electron Eng 13(5):365-375

36. Palreddy S, Zaghloul Al (2013) Circuit analysis of electromagnetic band gap (EBG) structures. In: Proceedings of the international symposium of electromagnetic theory, pp.67-70

37. Yang Guo, Xiang-Hua Wang, Jun Hu (2014) A new parallel meshing technique integrated into the conformal FDTD method for solving complex electromagnetic problems. Front Inf Technol Electron Eng 15(12):1087-1097

38. Mahajan RC, Vyas V (2018) Experimental review of microstrip antenna based shapes of EBG cells and gap width. J Adv Res Dyn Control Syst 10(7):210-219

39. Mahajan RC, Parashar V, Vyas V (2019) Modified unit cell analysis approach for EBG structure analysis for gap width study effect. In: Proceedings of the Third international conference on microelectronics, computing and communication systems. Springer Lecture Notes in Electrical Engineering, vol 556

40. Mahajan RC, Vyas V, Sutaone MS (2020) Performance prediction of electromagnetic band gap structure for microstrip antenna using FDTD-PBC unit cell analysis and Taguchi's multi-objective optimization method. Microelectr Eng J 219

41. Mahajan RC, Parashar V, Vyas V, Sutaone M (2019) Study and experimentation of defected ground surface and its implementation with transmission line. SN Appl Sci. https://doi. org/10.1007/s42452-019-0245-6

Publisher's Note Springer Nature remains neutral with regard to jurisdictional claims in published maps and institutional affiliations. 